\title{
BMJ Open Intravesical administration of combined hyaluronic acid (HA) and chondroitin sulfate (CS) for the treatment of female recurrent urinary tract infections: a European multicentre nested case-control study
}

\author{
Oriana Ciani, ${ }^{1,2}$ Erik Arendsen, ${ }^{3}$ Martin Romancik, ${ }^{4}$ Richard Lunik, ${ }^{5}$ \\ Elisabetta Costantini, ${ }^{6}$ Manuel Di Biase, ${ }^{6}$ Giuseppe Morgia, ${ }^{7}$ Eugenia Fragalà, ${ }^{7}$ \\ Tomaskin Roman, ${ }^{8}$ Marian Bernat, ${ }^{9}$ Giorgio Guazzoni, ${ }^{10}$ Rosanna Tarricone, ${ }^{1,11}$ \\ Massimo Lazzeri ${ }^{10}$
}

To cite: Ciani 0 , Arendsen $\mathrm{E}$, Romancik M, et al. Intravesical administration of combined hyaluronic acid (HA) and chondroitin sulfate (CS) for the treatment of female recurrent urinary tract infections: a European multicentre nested case-control study. BMJ Open 2016;6:e009669. doi:10.1136/bmjopen-2015009669

- Prepublication history and additional material is available. To view please visit the journal (http://dx.doi.org/ 10.1136/bmjopen-2015009669).

Received 10 August 2015 Revised 20 January 2016 Accepted 1 March 2016

CrossMark

For numbered affiliations see end of article.

Correspondence to

Dr Oriana Ciani;

oriana.ciani@unibocconi.it

\section{ABSTRACT}

Objectives: To compare the clinical effectiveness of the intravesical administration of combined hyaluronic acid and chondroitin sulfate $(\mathrm{HA}+\mathrm{CS})$ versus current standard management in adult women with recurrent urinary tract infections (RUTIs).

Setting: A European Union-based multicentre, retrospective nested case-control study.

Participants: 276 adult women treated for RUTIs starting from 2009 to 2013.

Interventions: Patients treated with either intravesical administration of $\mathrm{HA}+\mathrm{CS}$ or standard of care (antimicrobial/immunoactive prophylaxis/probiotics/ cranberry).

Primary and secondary outcome measures: The primary outcome was occurrence of bacteriologically confirmed recurrence within 12 months. Secondary outcomes were time to recurrence, total number of recurrences, health-related quality of life and healthcare resource consumption. Crude and adjusted results for unbalanced characteristics are presented.

Results: 181 patients treated with $\mathrm{HA}+\mathrm{CS}$ and 95 patients treated with standard of care from 7 centres were included. The crude and adjusted ORs $(95 \% \mathrm{Cl})$ for the primary end point were 0.77 (0.46 to 1.28) and 0.51 (0.27 to 0.96 ), respectively. However, no evidence of improvement in terms of total number of recurrences (incidence rate ratio $(95 \% \mathrm{Cl}), 0.99(0.69$ to 1.43)) or time to first recurrence was seen (HR $(95 \% \mathrm{Cl}), 0.99$ (0.61 to 1.61)). The benefit of intravesical $\mathrm{HA}+\mathrm{CS}$ therapy improves when the number of instillations is $\geq 5$.

Conclusions: Our results show that bladder instillations of combined $\mathrm{HA}+\mathrm{CS}$ reduce the risk of bacteriologically confirmed recurrences compared with the current standard management of RUTIs. Total incidence rates and hazard rates were instead nonsignificantly different between the 2 groups after

\section{Strengths and limitations of this study}

- These real-world data show that bladder instillations of combined hyaluronic acid and chondroitin sulfate may reduce the risk of bacteriologically confirmed urinary tract infections versus current standard management.

- However, if the recurrence occurs, there is no evidence of benefit in terms of total number or time to first recurrence.

- The number of instillations seems to be an important marker of success for this nonantimicrobial therapy.

- Owing to the retrospective observational design, these findings need confirmation from prospective and preferably randomised studies.

adjusting for unbalanced factors. In contrast to what happens with antibiotic prophylaxis, the effectiveness of the HA+CS reinstatement therapy improves over time.

Trial registration number: NCT02016118.

\section{BACKGROUND}

Urinary tract infection (UTI) is a major healthcare concern in women with an annual incidence of 30 per $1000 .^{1}$ Nearly $33 \%$ of women will have had at least one UTI episode, with characteristics of acute cystitis, requiring antimicrobial therapy by the age of 24 years and as many as $60 \%$ of women reporting having had a UTI in their lifetime. ${ }^{2} 3$

UTIs have a propensity to recur; ${ }^{4}{ }^{5}$ evidence shows that between $24 \%$ and $50 \%$ of 
initial episodes are followed by a second infection within 6 months. ${ }^{6-9}$ A widely accepted definition of recurrent UTIs (RUTIs) is two or more UTI episodes over 6 months, or three or more episodes over 12 months. ${ }^{10}$ On a population scale, the high incidence and prevalence of RUTIs results in considerable healthcare costs; at the individual level, the impact of this condition on health-related quality of life (HRQoL) is not negligible. ${ }^{11-13}$

The pathogenesis of RUTI involves colonisation of the vagina with uropathogenic bacteria and subsequent migration per urethra to the bladder. About $68-77 \%$ of recurrences caused by Escherichia coli involve strains genetically indistinguishable from those that caused previous infections. ${ }^{14}$

The diagnosis is often made on clinical presentation with local genitourinary symptoms of dysuria, frequency, and urgency or hesitancy appearing suddenly. ${ }^{14}$ However, urine culture is useful in women presenting with RUTI to confirm the diagnosis, direct antimicrobial therapy and exclude infection from an overactive bladder or interstitial cystitis. ${ }^{15}$ Evidence-based clinical practice guidelines recommend empiric initial therapy for acute management or continuous antimicrobial therapy or self-initiated therapy and prophylaxis, either antimicrobial or non-antimicrobial based. ${ }^{16} 17$

The choice of specific strategy for care depends on the number of recurrences experienced per year, the patient's preferences and careful review of modifiable risk factors. ${ }^{1418}$ As the second most common reason for prescribing antibiotics (following otitis media), there is currently increasing concern about empiric use of these agents due to increased antimicrobial resistance (AMR). Antibiotic use selects for resistant pathogens: a major risk factor for an antibioticresistant UTI is prior antibiotic use. ${ }^{5}$ In an international survey investigating the prevalence and susceptibility of pathogens causing cystitis, $10.3 \%$ of $E$. coli isolates were resistant to at least three different classes of antimicrobial agents, including ampicillin (48.3\%), trimethoprim/sulfamethoxazole $(29.4 \%)$ and nalidixic acid (18.6\%). ${ }^{19}$

Non-antimicrobial prevention strategies have become popular in the age of increasing antimicrobial use and resistance. However, no probiotic agent has been approved for therapeutic use and the potential benefit of cranberry in terms of product type (solid vs liquid), dosing and optimal patient population remains to be elucidated. ${ }^{14}{ }^{18}$ A new therapy based on the reinstatement of the glycosaminoglycan (GAG) bladder epithelium has recently been proposed for the treatment of RUTIs. ${ }^{20}$ This GAG layer consists of non-sulfated, for example, hyaluronic acid (HA), and sulfated, for example, heparan sulfate and heparin, chondroitin sulfate (CS), dermatan sulfate and keratan sulfate, GAGs. Limited evidence has shown the preventive activity of intravesical GAG substation therapy (with HA alone or with $\mathrm{HA}+\mathrm{CS}$ ) on recurrence of infections in patients with recurrent bacterial cystitis. ${ }^{21}$ However, large-scale studies are needed to underline the benefit of this therapy. ${ }^{22}$

Therefore, we decided to perform a European retrospective multicentre study to compare the clinical effectiveness of the intravesical administration of combined $\mathrm{HA}+\mathrm{CS}$ (ialuril, IBSA Institut Biochimique SA) versus current standard management of RUTIs in adult women.

\section{METHODS}

\section{Study design}

This was a European Union (EU) based multicentre, retrospective nested case-control comparison of individual patient data collected from electronic medical records and/or administrative databases available at the participating institutions. Centres using the intravesical administration of combined $\mathrm{HA}+\mathrm{CS}$, in the countries where ialuril was already registered and on the market (ialuril received a CE mark for this indication in 2009), were identified and invited to take part in the study.

\section{Study population}

All patients treated with either HA+CS or standard of care at the participating centres, high volume organisations with specific expertise in the treatment of UTIs, starting from 2009, were included if they were women, aged 18-75 years, diagnosed with RUTIs, defined as at least three episodes of uncomplicated UTIs accompanied by clinical symptoms and documented by urine culture with the isolation of $>10^{3} \mathrm{CFU} / \mathrm{mL}$ of an identified pathogen in the past 12 months. Uncomplicated UTI is defined as an infection in a person with a normal urinary tract and function. ${ }^{17}$ Women with complicated UTIs (ie, individuals with functional or structural abnormalities of the genitourinary tract) were excluded. Within Europe, patients at first diagnosis of RUTIs are offered an approach based on behavioural changes, antimicrobial prophylaxis or aspecific non-antimicrobial prevention. However, several women refuse to take antimicrobials over an extended period of time; hence, intravesical administration of $\mathrm{HA}+\mathrm{CS}$ is intended for women refractory or not satisfied with first-line management of RUTIs. On the basis of a previous cohort study, ${ }^{11}$ we estimated that 208 patients were needed to observe a $50 \%$ difference in the proportions of patients recurring between the two groups within 12 months with $90 \%$ power and an $\alpha$-level of 0.05 .

\section{Groups and interventions}

Patients were treated with intravesical administration of combined HA $1.6 \%$ and CS $2.0 \%$. The recommended scheme is one instillation per week for the first month, followed by one instillation every 2 weeks for the second month and one instillation per month afterwards until stable remission of the symptoms; however, different patterns are seen in clinical practice. These patients were compared with patients treated with antimicrobial 
prophylaxis (continuous or postcoital), or immunoactive prophylaxis or prophylaxis with probiotics or prophylaxis with cranberry, or a combination of these, ${ }^{17}$ as recommended by the European Association of Urology.

\section{Study outcomes}

The primary outcome for this study was the occurrence of objective UTI recurrence, defined as the occurrence of at least one bacteriologically confirmed UTI within 12 months after treatment initiation for RUTIs. According to current clinical guidelines, in nonpregnant women, urine culture is recommended in symptomatic patients only. Information about clinically confirmed recurrences was also sought, although they are not reported in this manuscript as they are assumed to be less objective than the bacteriologically confirmed ones. Patients who developed a UTI while on the $\mathrm{HA}+\mathrm{CS}$ instillation protocol were treated according to clinical guidelines with antibiotics but could continue the instillations afterwards. After the first bacteriologically confirmed recurrence, the time to first recurrence was recorded, as well as the number of additional UTIs. The secondary outcome measures were the time to recurrence (defined as the time from the start of the treatment until the occurrence of the first objective recurrence); the total number of recurrences; HRQoL as assessed through the Short Form 36 (SF-36) ${ }^{23}$ or Euro QoL 5D $(\mathrm{EQ}-5 \mathrm{D})^{24}$ questionnaires. Dutch, ${ }^{25}$ Italian $^{26}$ and $\mathrm{UK}^{27}$ tariffs were used to estimate utility values from the EQ-5D questionnaires in the Netherlands, Italy and Slovakia, respectively. Information about healthcare resource consumption was also collected. A cost analysis was planned and will be the subject of a future publication.

\section{Data collection}

General patient demographic characteristics, diagnosis and treatment information were collected on the basis of a predefined form designed on the input obtained from collaborating centres during a workshop held in July 2013. An intuitive electronic system was implemented (Advice Pharma Ltd) to record and store data on a secure remote server provider.

\section{Statistical analyses}

Continuous baseline characteristics are presented as the median and IQR or mean and SD, as appropriate. For proportions, absolute and relative frequencies are reported. The Wilcoxon-Mann-Whitney test or Student $\mathrm{t}$ test was used for continuous and ordinal variables baseline differences, whereas the $\chi^{2}$ test was used for proportions. In our primary analyses, we applied logistic, Poisson and Cox regression for objective recurrence, number of recurrences and time to recurrence, respectively. Results were presented as crude and adjusted OR, incidence rate ratio (IRR) and HR, respectively, with their $95 \%$ CIs. Adjusting variables were age, body mass index (BMI), employment and menopause status, postcoital infections, dyspareunia, Female Sexual Function
Index (FSFI) and severity of RUTI. A prespecified sensitivity analysis was conducted to investigate the impact of adherence to HA+CS treatment on clinical outcomes considering patients who had $\geq 5$ instillations. Pairwise deletion was used to deal with missing data. All significance tests were two-tailed at the 0.05 significance level. All the analyses were conducted using Stata SE StataCorp LP 11.

\section{RESULTS}

Overall, 276 patients treated for RUTIs at seven European centres from January 2009 up to December 2013 were included in the analyses. Of these, 181 women were treated with HA+CS intravesical administration and 95 women received standard management of RUTIs. The numerical imbalance was probably due to the participating organisations being tertiary referral centres for patients who are not satisfied with standard management of RUTIs. A flow diagram reporting the number of patients at each stage of the study is shown in figure 1. The baseline sociodemographic and clinical characteristics of patients are reported in table 1. Given the non-experimental nature of the study, the distribution of several characteristics was not homogeneous between the two groups; in particular, women treated with $\mathrm{HA}+\mathrm{CS}$ were older, with a higher BMI and probability of dyspareunia.

\section{Primary analyses}

In the HA+CS group, $55.7 \%$ of patients showed bacteriologically confirmed recurrences, whereas $62.1 \%$ had such recurrence in the standard of care group $(\mathrm{p}=0.313)$. However, the adjusted OR (95\% CI) for developing a bacteriologically confirmed recurrence within 12 months was 0.51 ( 0.27 to 0.96$)$, meaning that, other characteristics being equal, there is a $49 \%$ reduced risk of developing a recurrence in patients treated with HA+CS compared with standard care (table 2). When the number of recurrences is considered, in the $\mathrm{HA}+\mathrm{CS}$ group there were 121 bacteriologically confirmed recurrences in 61.5

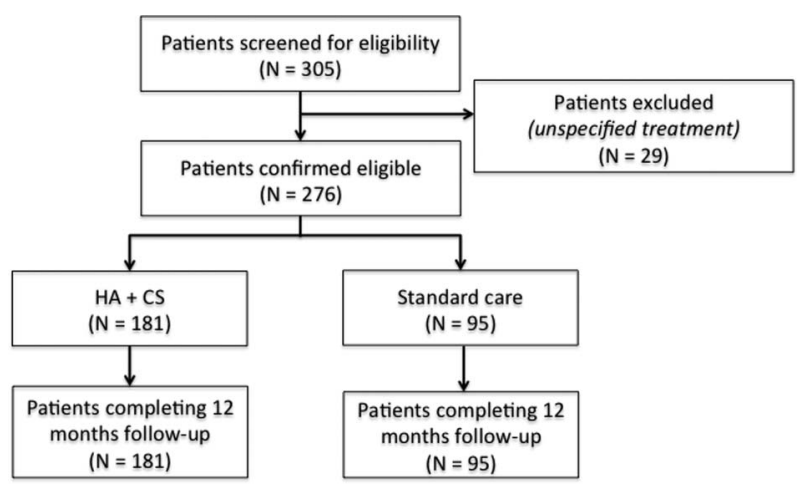

Figure 1 Flow diagram describing numbers of individuals at each stage of study. $\mathrm{HA}+\mathrm{CS}$, hyaluronic acid and chondroitin sulfate. 
Table 1 Baseline characteristics

\begin{tabular}{|c|c|c|c|}
\hline Characteristics & $\mathrm{HA}+\mathrm{CS}(\mathrm{N}=181)$ & Standard care $(\mathrm{N}=95)$ & p Value \\
\hline Age-mean (SD) & $55.24(17.33)$ & $48.84(21.16)$ & 0.017 \\
\hline Employed-n (\%) & $83(46)$ & $62(65)$ & 0.003 \\
\hline Partner-n (\%) & $148(82)$ & $72(76)$ & 0.172 \\
\hline Sexually active $-n(\%)$ & $114(63)$ & $60(63)$ & 0.931 \\
\hline Menopause-n (\%) & $69(28)$ & $49(51)$ & 0.038 \\
\hline BMI-mean (SD) & $26.20(6.37)$ & $24.56(5.03)$ & 0.019 \\
\hline Postcoital infections-n (\%) & $31(17)$ & $29(31)$ & 0.012 \\
\hline Dyspareunia—n (\%) & $40(22)$ & $5(5)$ & $<0.001$ \\
\hline Severity RUTI-median (IQR)* & $4(2-5)$ & $4(3-4)$ & 0.316 \\
\hline \multicolumn{4}{|l|}{ Prophylaxis-n (\%) } \\
\hline Antimicrobial prophylaxis continuous & NA & $42(44.21)$ & \\
\hline Antimicrobial prophylaxis postcoital & NA & $19(20)$ & \\
\hline Intermittent immunoactive prophylaxis & NA & $28(29.47)$ & \\
\hline On demand immunoactive therapy & NA & $1(1.05)$ & \\
\hline Others & NA & $5(5.26)$ & \\
\hline FSFI-mean (SD) & $5.35(8.71)$ & $3.13(5.43)$ & 0.018 \\
\hline EQ-5D—median (IQR) & $0.69(0.24-0.73) \dagger$ & $0.69(0.28-0.81) \ddagger$ & 0.696 \\
\hline SF-36 PCS-median (IQR) & $60(54.5-70) \S$ & $60(53-67.5) \emptyset$ & 0.500 \\
\hline SF-36 MCS-median (IQR) & $60(54-70)^{\star \star}$ & $60(53-67.5) \uparrow$ & 0.551 \\
\hline
\end{tabular}

${ }^{*}$ According to the European Association of Urology Guidelines on Urological Infections where 1 is low severity cystitis and 6 is extreme severity including organ failure. ${ }^{21}$

$\dagger \mathrm{N}=90$ patients.

$\ddagger \mathrm{N}=29$ patients.

$\S \mathrm{N}=72$ patients.

$\mathbb{7 N}=60$ patients.

${ }^{\star \star} \mathrm{N}=73$ patients.

BMI, body mass index; EQ-5D, Euro QoL 5D 3 level; FSFI, Female Sexual Function Index; HA+CS, hyaluronic acid and chondroitin sulfate; NA, not applicable; RUTI, recurrent urinary tract infection; SF-36 MCS, Short Form 36 mental component score; SF-36 PCS, Short Form 36 physical component score.

person-years, whereas in the standard treatment group there were 59 bacteriologically confirmed recurrences in 51.1 person-years $(\mathrm{p}=0.001)$. However, we observed an adjusted IRR (95\% CI) of 0.99 (0.69 to 1.43), showing non-significant differences in the incidence rates between the group treated with HA+CS and the control. Similar results were obtained from the univariate and multivariate Cox regression models used to estimate the HR $(95 \%$ CI) for the time to first bacteriologically confirmed recurrence, with an unadjusted estimate of 0.99 (0.61 to 1.61) (table 2). Although the median time to first recurrence was higher in the standard care group (169.5 days (IQR, 72.5-341.5) vs 320 days (IQR,
179-365); $\mathrm{p}$ value <0.001) during the 12-month follow-up, we observed the distribution of recurrences at separate follow-up times, and noted that the incident proportion of patients who developed the recurrence versus those still at risk was lower in the HA+CS group in the latest part of follow-up, after 8 months (table 3). All patients were alive at the 12-month follow-up. There were 14 all-cause hospitalisations in the HA+CS group and 1 in the control group.

\section{HRQOL and resources consumption}

In a subset of patients, a measure of the HRQoL as measured through the SF-36 or EQ-5D 3 level questionnaires

Table 2 Bacteriologically confirmed recurrence, total number of recurrences and time to first recurrence between $\mathrm{HA}+\mathrm{CS}$ versus standard of care treated patients

\begin{tabular}{|c|c|c|}
\hline Outcome & OR $(95 \% \mathrm{Cl})$ & Adjusted* OR (95\% Cl) \\
\hline \multirow[t]{2}{*}{ Bacteriologically confirmed recurrence } & $0.77(0.46$ to 1.28$)$ & $0.51(0.27$ to 0.96$)$ \\
\hline & IRR $(95 \% \mathrm{CI})$ & Adjusted* IRR (95\% Cl) \\
\hline \multirow[t]{2}{*}{ Total number of bacteriologically confirmed recurrence } & $1.73(1.27$ to 2.37$)$ & $0.99(0.69$ to 1.43$)$ \\
\hline & HR (95\% Cl) & Adjusted* $\mathrm{HR}(95 \% \mathrm{Cl})$ \\
\hline Time to first bacteriologically confirmed recurrence & $1.66(1.09$ to 2.54$) \dagger$ & $0.99(0.61$ to 1.61$)$ \\
\hline
\end{tabular}


Table 3 Incidence of bacteriologically confirmed recurrences during 12-month follow-up

\begin{tabular}{llll}
\hline $\begin{array}{l}\text { Incidence of } \\
\text { bacteriologically } \\
\text { confirmed recurrences } \\
\text { (days) }\end{array}$ & $\begin{array}{l}\text { HA } \\
+ \text { +CS } \\
\text { (\%) }\end{array}$ & $\begin{array}{l}\text { Standard } \\
\text { care (\%) }\end{array}$ & $\begin{array}{l}\mathbf{p} \\
\text { Value }\end{array}$ \\
\hline $0-90$ & 15.3 & 12.7 & 0.610 \\
$91-180$ & 15.6 & 12.9 & 0.628 \\
$181-240$ & 10.7 & 3.7 & 0.132 \\
$241-365$ & 16.3 & 30.8 & 0.043 \\
\hline
\end{tabular}

$\mathrm{HA}+\mathrm{CS}$, hyaluronic acid and chondroitin sulfate.

was available at baseline and after 12 months of follow-up. There was no evidence of better improvement in HRQoL in the HA+CS group compared with control with SF-36 results, whereas when EQ-5D data were considered, the HA+CS group seemed to have received a higher benefit in terms of HRQoL than the control (see online supplementary table $\mathrm{S} 1$ ).

There is a general reduction in physical units of health resources (ie, medical visits, laboratory and imaging tests) consumed by the two groups before the treatment and during the follow-up (see online supplementary table S2) without significant differences between the two groups.

\section{Sensitivity analyses}

We repeated all primary analyses and considered different exposure intensity (ie, number of intravesical administrations received) in the $\mathrm{HA}+\mathrm{CS}$ group. All findings consistently show the additional benefit gained by patients when the number of instillations increases, possibly revealing the importance of adherence to this medical device therapy for the treatment of RUTIs (table 4). As a post hoc subgroup analysis, we repeated primary analyses in non-sexually active patients only and obtained similar patterns of results as in the whole sample, although with loss of statistical significance.

\section{DISCUSSION}

In this European multicentre retrospective observational study, we compared bacteriologically confirmed recurrence rates at the 12 month follow-up after the initiation of intravesical administration of $\mathrm{HA}+\mathrm{CS}$ versus standard of care for the treatment of RUTIs. After adjusting for unbalanced confounding factors between the two groups, we observed that the $\mathrm{HA}+\mathrm{CS}$ patients had a $49 \%$ reduction (OR $0.51,95 \%$ CI 0.27 to 0.96 ) in the risk of a bacteriologically confirmed recurrence, whereas there was no statistical evidence for a difference in the incidence and hazard rates of such recurrences between the two groups.

Four clinical studies ${ }^{28-31}$ have been performed to investigate the efficacy and tolerability of intravesically administered GAG for RUTI prophylaxis, all showing that $\mathrm{HA}$ alone or $\mathrm{HA}+\mathrm{CS}$ instillations reduce the number of UTIs per patient per year at no increased risk of severe adverse events and prolong the time interval between RUTI episodes, with a high rate of patients being free of recurrence at the end of the study period. In particular, two randomised control trials (RCTs) studies compared $\mathrm{HA}+\mathrm{CS}$ administration to either placebo $^{29}$ or long-term antibiotic prophylaxis using sulfamethoxazole $200 \mathrm{mg}$ and trimethoprim $40 \mathrm{mg} .^{30}$ Damiano et al report a decrease in the UTI rate per patient of $77 \%$ (95\% CI 72.3 to 80.8 ) in the experimental versus placebo group, whereas De Vita and colleagues report the mean $\pm \mathrm{SD}$ number of recurrent cystitis per patient per year as $1 \pm 1.2$ vs $2.3 \pm 1.4$ in $\mathrm{HA}+\mathrm{CS}$ and antibiotic treated patients, respectively. Despite the prospective and randomised design, these trials were limited by the small sample size (ie, they included 57 and 28 patients, respectively) and the single centre setting that considerably reduces the generalisability of

Table 4 Sensitivity analysis-impact of number of intravesical administration of HA+CS on clinical outcomes

\begin{tabular}{|c|c|c|}
\hline Bacteriologically confirmed recurrence & OR $(95 \% \mathrm{Cl})$ & Adjusted* OR (95\% Cl) \\
\hline$\geq 5$ Instillations $(n=156)$ vs standard of care & $0.81(0.48$ to 1.36$)$ & $0.52(0.27$ to 0.99$)$ \\
\hline$\geq 6$ Instillations $(n=134)$ vs standard of care & $0.69(0.40$ to 1.18$)$ & $0.47(0.25$ to 0.91$)$ \\
\hline$\geq 7$ Instillations $(n=82)$ vs standard of care & $0.63(0.34$ to 1.14$)$ & $0.43(0.21$ to 0.88$)$ \\
\hline Total number of bacteriologically confirmed recurrence & IRR $(95 \% \mathrm{CI})$ & Adjusted* IRR (95\% Cl) \\
\hline$\geq 5$ Instillations vs standard of care & $1.82(1.33$ to 2.49$)$ & $1.05(0.73$ to 1.52$)$ \\
\hline$\geq 6$ Instillations vs standard of care & $1.64(1.18$ to 2.28$)$ & $0.97(0.66$ to 1.40$)$ \\
\hline$\geq 7$ Instillations vs standard of care & 1.46 (1 to 2.13$)$ & 0.90 (0.60 to 1.36$)$ \\
\hline Time to first bacteriologically confirmed recurrence & HR $(95 \% \mathrm{CI})$ & Adjusted* HR (95\% Cl) \\
\hline$\geq 5$ Instillations vs standard of care & $1.72(1.12$ to 2.65$)$ & $1.04(0.64$ to 1.71$)$ \\
\hline$\geq 6$ Instillations vs standard of care & 1.55 (0.99 to 2.41$)$ & $0.96(0.58$ to 1.57$)$ \\
\hline$\geq 7$ Instillations vs standard of care & 1.33 (0.79 to 2.22$)$ & $0.85(0.49$ to 1.47$)$ \\
\hline
\end{tabular}


the findings. While waiting for definitive RCT evidence clarifying the comparative effectiveness profile of this therapy in support of its adoption, our observational study design provides useful information around its effectiveness in real-world practice.

In this respect, our study involved seven centres across three European countries and 276 patients, thus providing important additional evidence with respect to current treatment options for RUTIs. Furthermore, the non-experimental observational design allows for a closer representation of the routine clinical practice of the use of the HA+CS reinstatement therapy as compared with standard of care in place at high volume university hospitals, that is on purpose defined as very broad given the variety of recommended strategies ${ }^{17}$ and general scarce adherence to clinical guidelines. ${ }^{32}$

On the other hand, the retrospective design limited the availability of data to that previously collected at the centres. Contacting patients ex-post to gather additional data was not applicable (eg, as in the case of HRQoL assessment) or not helpful, given the potential significant recall bias introduced by delayed reporting. The issue of missing data was dealt with by assuming that they were missing at random (ie, given the observed data, data are missing independently of unobserved data, ie, missing data do not depend on the level of their outcome) and applying pairwise deletion. In this regard, we performed two additional analyses, first by restricting the primary analyses to all-complete-cases (ie, no missing values in outcomes and adjusting variables) and providing similar results to those presented here (data not shown). Second, for all outcomes and adjusting variables, we tested through Fisher's exact test whether proportions of missing values was different between $\mathrm{HA}+\mathrm{CS}$ and Standard of Care groups. No significant difference was observed with the exception of the resource consumption where the number of missing values was higher in the $\mathrm{HA}+\mathrm{CS}$ group.

Data on uropathogens and AMR within the groups were unfortunately not available from this database, although we know that the most commonly prescribed antibiotics were ciprofloxacin (13.2\% of all prescriptions), cefuroxime $(6.9 \%)$, fosfomycin $(6.9 \%)$, nitrofurantoin $(6.4 \%)$ and E. coli bacterial extract (OM-89, 4.8\%).

HRQoL assessment in routine practice is still uncommon, as indicated by the significant proportion of missing information (up to $73 \%$ in the control group) on this outcome. However, our findings are in line with previous reports showing that the GAG replacement treatment in women with RUTIs had a positive impact on patients' quality of life, reducing the symptoms and improving the maximum cystometric capacity. ${ }^{29} 30$

RUTIs in women are a common condition, associated with significant morbidity and burden for the whole society. In a study of 684 women aged $18-70$ years with UTI, participants reported an average of 3.83 symptom days, 2.89 restricted-activity days, and 3.13 days during which they were unwell. ${ }^{33}$ In another study, patients reported 1.2 days on which they were not able to attend classes or work, and 0.4 days in bed. ${ }^{34}$ New effective prevention strategies are needed; in particular, nonantimicrobial approaches would be desirable for several reasons. First of all, a prolonged antibiotic use as a prophylactic approach to RUTI increases the risk of side effects, including vaginal and oral candidiasis, and gastrointestinal symptoms. ${ }^{10}$ This, in turn, lowers patients' compliance and therefore the effectiveness of the treatment. ${ }^{35}$ However, the most worrying effect of the antibiotic use (and misuse) is the exacerbation of AMR. ${ }^{36}$ Recently, a UK commissioned report on health and macroeconomic consequences of AMR estimated 10 million extra deaths a year and costs up to $€ 90$ trillion for the global economy by 2050 if this problem is not tackled properly. ${ }^{37}$ Although this first report might not be as scientifically rigorous or informed by evidence as possible ${ }^{38}$ it brought renewed interest in the worldwide AMR crisis. The war against the spread of drug-resistant microbes is attracting considerable attention as well as investment from all major governments and research organisations in the EU and beyond. ${ }^{39} 40$ The way forward outlined by all of these major research initiatives includes establishing appropriate funding and rewards to subsidise access to and development of new antibiotic agents, preservation of existing drugs antimicrobial activity through prescription tailored to diagnosis, prioritisation and controlled access, and identification of novel approaches and therapies for microbial diseases.

The case of GAG reinstatement therapy is a good example of an innovative approach to prevent and manage bacterial urinary infections, a medical device intervention as opposed to a drug. In contrast to antibiotic therapy, which aims at eradicating pathogens, treatment with $\mathrm{HA}+\mathrm{CS}$ targets bacterial adherence to the bladder mucosa by physically recovering a damaged GAG layer that facilitates bacterial adherence and, therefore, RUTIs. Although patients who benefit from the treatment in the first place might decide to undertake a higher number of instillations compared with patients who do not benefit immediately, the different mechanism of action could explain the apparent reduction in the incidence of UTIs in the group treated with HA+CS instillations compared with standard care when considering later time intervals (table 3). While antibiotics are immediately effective, although subject and conducive to resistance, GAG layer administration is progressively restoring the epithelium that will protect women from future uropathogen infections. On the other hand, catheterisationinduced UTIs might represent an unintended consequence of this procedure. Previous reports ${ }^{29}{ }^{30}$ have highlighted good tolerability and safety of the intervention that must be performed under sterile conditions by nurses trained in the procedure. As regards the economic profile of the two alternative approaches, it has been reported that the cost of $\mathrm{HA}+\mathrm{CS}$ could be even five times higher than the cost for a 6-month antibiotic prophylaxis. However, this consideration corresponds to a very 
restrictive, if not naïve, cost-analysis as it is well known that all direct healthcare costs and consequences, including those for the wider society, as would be containment of drug-resistance spreading, should be taken into account when assessing the cost-effectiveness profiles of health technologies. Future methodologically sound economic evaluation studies are recommended to compare the societal or payer value of the two treatment strategies.

\section{CONCLUSIONS}

In order to treat and prevent RUTI, there is a need for effective and safe alternative strategies for antimicrobial therapy. Our study showed that in a real-world setting, bladder instillation of combined HA+CS may reduce the risk of bacteriologically confirmed recurrences compared with the current standard management in this study population. Total incidence rates and hazard rates were instead non-significantly different between the two groups. The number of HA+CS instillations seems to be an important marker of success for intravesical administration therapy. Furthermore, in contrast to what happens with antibiotic prophylaxis, owing to side effects and development of resistance, the effectiveness of GAG reinstatement therapy improves over time, with an even better expected comparative effectiveness profile in the long run.

Although firm conclusions are difficult due to the retrospective observational design, these findings highlight the relevance of additional prospective and randomised studies in this area and the promising role of the HA+CS reinstatement therapy for prevention and treatment of RUTI in an era of worryingly increased AMR.

\section{Author affiliations}

${ }^{1}$ Centre for Research on Health and Social Care Management, Università Bocconi, Milan, Italy

${ }^{2}$ Evidence Synthesis \& Modelling for Health Improvement, Institute of Health Research, University of Exeter Medical School, Exeter, UK

${ }^{3}$ Diaconessenhuis, Maatschap, Urologie, Leiden, The Netherlands

${ }^{4}$ Department of Urology, St. Cyril and Method University Hospital, Bratislava, Slovakia

${ }^{5}$ Department of Urology, Fakultná nemocnica s poliklinikou, Prešov, Slovakia

${ }^{6}$ Department of Surgical and Biomedical Science, University of Perugia,

Urology and Andrology Clinic, Perugia, Italy

${ }^{7}$ Department of Urology, University of Catania, Catania, Italy

${ }^{8}$ Department of Urology, Jessenius School of Medicine, University Hospital, Martin, Slovakia

${ }^{9} \mathrm{FNsP}$, Urologicka Klinika, Nové Zámky, Slovakia

${ }^{10}$ Department of Urology, Humanitas Clinical and Research Center, Milan, Italy

${ }^{11}$ Department of Policy Analysis and Public Management, Università Bocconi, Milano, Italy

Twitter Follow Oriana Ciani at @OrianaCiani

Contributors $\mathrm{OC}, \mathrm{RT}$ and $\mathrm{ML}$ designed the study. EA, MR, RL, EC, MDB, GM, $\mathrm{EF}, \mathrm{TR}, \mathrm{MB}$ and $\mathrm{GG}$ contributed to the data collection. $\mathrm{OC}$ analysed the data and drafted the manuscript. All the authors commented on and approved the final version of the manuscript.

Funding This study was funded by an unrestricted grant from the TETI Association-study group for urogenital diseases. Members of the association were involved in the data collection and revised the manuscript.

Competing interests None declared.
Ethics approval The study protocol was reviewed and approved at the coordinating centre by an Independent Ethics Committee at the Department of Urology, University of Perugia.

Provenance and peer review Not commissioned; externally peer reviewed.

Data sharing statement The data set is available by emailing the corresponding author.

Open Access This is an Open Access article distributed in accordance with the Creative Commons Attribution Non Commercial (CC BY-NC 4.0) license, which permits others to distribute, remix, adapt, build upon this work noncommercially, and license their derivative works on different terms, provided the original work is properly cited and the use is non-commercial. See: http:// creativecommons.org/licenses/by-nc/4.0/

\section{REFERENCES}

1. Laupland KB, Ross T, Pitout JD, et al. Community-onset urinary tract infections: a population-based assessment. Infection 2007;35:150-3.

2. Foxman B. Epidemiology of urinary tract infections: incidence, morbidity, and economic costs. Am J Med 2002;113(Suppl 1A):5S-13S.

3. Foxman B, Barlow R, D'Arcy $\mathrm{H}$, et al. Urinary tract infection: self-reported incidence and associated costs. Ann Epidemiol 2000;10:509-15.

4. Hooton TM, Scholes D, Hughes JP, et al. A prospective study of risk factors for symptomatic urinary tract infection in young women. N Engl J Med 1996;335:468-74.

5. Foxman B. The epidemiology of urinary tract infection. Nat Rev Urol 2010;7:653-60.

6. Sen A. Recurrent cystitis in non-pregnant women. Clin Evid 2008;07:801.

7. Ikaheimo R, Siitonen A, Heiskanen T, et al. Recurrence of urinary tract infection in a primary care setting: analysis of a 1-year follow-up of 179 women. Clin Infect Dis 1996;22:91-9.

8. Foxman $B$. Recurring urinary tract infection: incidence and risk factors. Am J Public Health 1990;80:331-3.

9. Foxman B, Gillespie B, Koopman J, et al. Risk factors for second urinary tract infection among college women. Am J Epidemiol 2000;151:1194-205.

10. Albert X, Huertas I, Pereiró II, et al. Antibiotics for preventing recurrent urinary tract infection in non-pregnant women. Cochrane Database Syst Rev 2004;(3):CD001209.

11. Ciani O, Grassi D, Tarricone R. An economic perspective on urinary tract infection: the 'costs of resignation'. Clin Drug Investig 2013;33:255-61.

12. Renard J, Ballarini S, Mascarenhas T, et al. Recurrent lower urinary tract infections have a detrimental effect on patient quality of life: a prospective, observational study. Infect Dis Ther 2015;4:125-35.

13. Bermingham SL, Ashe JF. Systematic review of the impact of urinary tract infections on health-related quality of life. BJU Int 2012;110(Pt C):E830-6.

14. Gupta K, Trautner BW. Diagnosis and management of recurrent urinary tract infections in non-pregnant women. BMJ 2013;346:f3140.

15. Arya LA, Northington GM, Asfaw T, et al. Evidence of bladder oversensitivity in the absence of an infection in premenopausal women with a history of recurrent urinary tract infections. BJU Int 2012;110:247-51.

16. Gupta K, Hooton TM, Naber KG, et al. International clinical practice guidelines for the treatment of acute uncomplicated cystitis and pyelonephritis in women: a 2010 update by the Infectious Diseases Society of America and the European Society for Microbiology and Infectious Diseases. Clin Infect Dis 2011;52:e103-20.

17. Grabe M, Bartoletti R, Bjerklund Johansen $\mathrm{T}$, et al. Guidelines on urological infections. Eur Assoc Urol 2015. http://uroweb.org/wpcontent/uploads/19-Urological-infections_LR2.pdf

18. Shepherd AK, Pottinger PS. Management of urinary tract infections in the era of increasing antimicrobial resistance. Med Clin North Am 2013;97:737-57, xii.

19. Schito GC, Naber KG, Botto H, et al. The ARESC study: an international survey on the antimicrobial resistance of pathogens involved in uncomplicated urinary tract infections. Int $J$ Antimicrob Agents 2009;34:407-13.

20. Lazzeri M, Montorsi F. The therapeutic challenge of "chronic cystitis": search well, work together, and gain results. Eur Urol 2011;60:78-80.

21. De Vita D, Antell H, Giordano S. Effectiveness of intravesical hyaluronic acid with or without chondroitin sulfate for recurrent 
bacterial cystitis in adult women: a meta-analysis. Int Urogynecol J 2013;24:545-52.

22. Madersbacher H, van Ophoven A, van Kerrebroeck PE. GAG layer replenishment therapy for chronic forms of cystitis with intravesical glycosaminoglycans-a review. Neurourol Urodyn 2013;32:9-18.

23. Brazier JE, Harper R, Jones NM, et al. Validating the SF-36 health survey questionnaire: new outcome measure for primary care. $B M J$ 1992;305:160-4.

24. EuroQol Group. EuroQol-a new facility for the measurement of health-related quality of life. Health Policy 1990;16:199-208.

25. Lamers LM, McDonnell J, Stalmeier PF, et al. The Dutch tariff: results and arguments for an effective design for national EQ-5D valuation studies. Health Econ 2006;15:1121-32.

26. Scalone L, Cortesi PA, Ciampichini R, et al. Italian population-based values of EQ-5D health states. Value Health 2013;16:814-22.

27. Dolan P. Modeling valuations for EuroQol health states. Med Care 1997:35:1095-108.

28. Constantinides C, Manousakas T, Nikolopoulos P, et al. Prevention of recurrent bacterial cystitis by intravesical administration of hyaluronic acid: a pilot study. BJU Int 2004;93:1262-6.

29. Damiano R, Quarto G, Bava I, et al. Prevention of recurrent urinary tract infections by intravesical administration of hyaluronic acid and chondroitin sulphate: a placebo-controlled randomised trial. Eur Urol 2011;59:645-51.

30. De Vita D, Giordano S. Effectiveness of intravesical hyaluronic acid/ chondroitin sulfate in recurrent bacterial cystitis: a randomized study. Int Urogynecol J 2012;23:1707-13.
31. Lipovac M, Kurz C, Reithmayr F, et al. Prevention of recurrent bacterial urinary tract infections by intravesical instillation of hyaluronic acid. Int J Gynaecol Obstet 2007;96:192-5.

32. Taur Y, Smith MA. Adherence to the Infectious Diseases Society of America guidelines in the treatment of uncomplicated urinary tract infection. Clin Infect Dis 2007;44:769-74.

33. Little $\mathrm{P}$, Merriman $\mathrm{R}$, Turner $\mathrm{S}$, et al. Presentation, pattern, and natural course of severe symptoms, and role of antibiotics and antibiotic resistance among patients presenting with suspected uncomplicated urinary tract infection in primary care: observational study. BMJ 2010;340:b5633.

34. Ronald A. The etiology of urinary tract infection: traditional and emerging pathogens. Am J Med 2002;113(Suppl 1A):14S-19S.

35. Hooton TM, Levy SB. Antimicrobial resistance: a plan of action for community practice. Am Fam Physician 2001;63:1087-98.

36. Fauci AS, Marston ID. The perpetual challenge of antimicrobial resistance. JAMA 2014;311:1853-4.

37. Review on Antimicrobial Resistance. Antimicrobial resistance: tackling a crisis for the health and wealth of nations. 2014. http:// amr-review.org/sites/default/files/AMR\%20Review\%20Paper\%20-\% 20Tackling\%20a\%20crisis\%20for\%20the\%20health\%20and\% 20wealth\%20of\%20nations_1.pdf

38. [No authors listed]. Antimicrobial resistance: in terms politicians understand. Lancet 2014;384:2173.

39. Woolhouse M, Farrar J. Policy: an intergovernmental panel on antimicrobial resistance. Nature 2014;509:555-7.

40. Geoghegan-Quinn M. Funding for antimicrobial resistance research in Europe. Lancet 2014;384:1186. 